\title{
Development of Novel Orifice Die Design for Precise Entrance Pressure Drop Measurements
}

\author{
Martin Zatloukal and Jan Musil \\ Polymer Centre, Faculty of Technology, Tomas Bata University in Zlin, TGM 275, Zlín 76272, \\ Czech Republic
}

\begin{abstract}
In this work, novel patent pending orifice die design for precise extensional viscosity data determination from entrance pressure drop measurements has been developed and tested both, theoretically (through Finite Element Analysis) and experimentally. It has been demonstrated that the proposed novel orifice die allows much more precise extensional viscosity measurements for polymer melts in comparison with conventionally used orifice dies.
\end{abstract}

Keywords: Orifice die, Entrance pressure drop, Extensional viscosity, Polymer melts.

PACS: 47.11.Fg, 47.50.Cd, 47.50.Ef, 83.10.Ff, 83.50.Ha, 83.50.Uv, 83.80.Sg, 83.85.Cg, 83.85.Rx

\section{INTRODUCTION}

Extensional viscosity is an important rheological property which expresses the resistance of the polymer melt against to the extensional flow (stretching). The knowledge of extensional viscosity is crucially important for better understanding of polymer molecular structure and it significantly helps to optimize the polymer processing conditions and equipment design to achieve stable flow conditions especially in technologies with predominantly extensional flows (the flow through converging channels in the extrusion/coextrusion dies, injection moulding, calendering and post die processes such as, fibber spinning, tubular film blowing, cast film). It should be mentioned that the extensional viscosity of the polymer melt is much more difficult to measure in comparison with the shear viscosity [1]. In the open literature, several types of extensional rheometers and experimental techniques have been developed [1-9] to measure this very important property. However each of them is applicable for only limited range of extensional rates or stresses and the low experimental error can be observed only in the case when the elongation flow is stable for a sufficient time for large enough volume of the sample. Interestingly, the only available experimental technique to determine extensional viscosity at high extensional strain rates is based on the entrance pressure drop measurements [1,5-9] by using capillary rheometers (see Figure 1). 


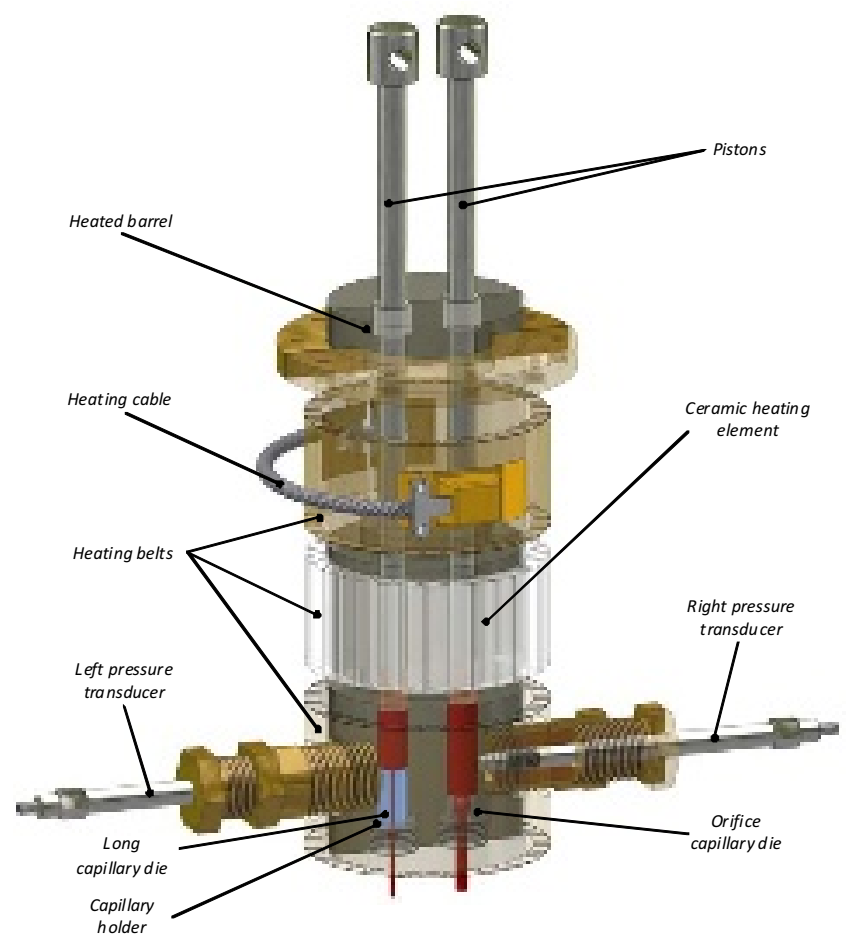

FIGURE 1. Transparent section view of barrel part of twin-bore capillary rheometer.

The use of an orifice die for entrance pressure drop measurements is very attractive methodology because it minimizes errors due to pressure dependence of viscosity, wall slip and viscous heating which can occur during alternative extrapolation based Bagley plot approach. On the other hand, Kim and Dealy [8] recently pointed out that entrance pressure drop measurements can be highly erroneous by using orifice dies for the cases when orifice die exit section is wetted by the polymer melt. With the aim to overcome this conventional orifice die weakness, the theoretical and experimental flow analysis has been performed to obtain novel improved orifice die design.

\section{Theoretical and Experimental Analysis}

\section{Theoretical Effect of Orifice Die Design on the Measured Extensional Rheology}

It may happen that, due to quite narrow conventional orifice die exit region (see Figure 2 for more detail) and extrudate swell, the polymer melt starts to fill this region. 


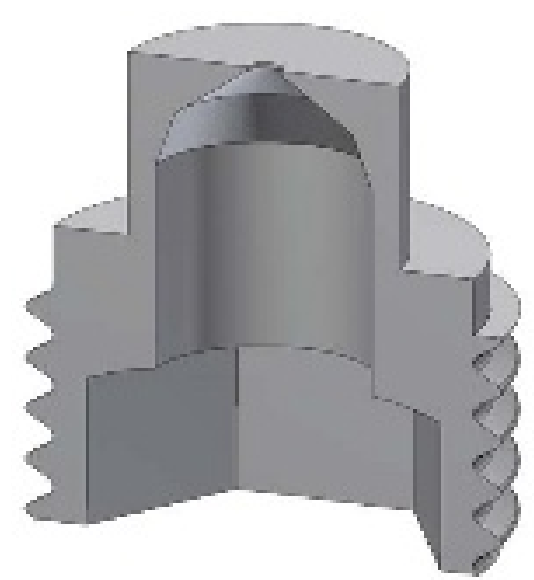

FIGURE 2. Conventional orifice die design.

Therefore, if this happens, the question is how much this flow situation influences the measured entrance viscosity/uniaxial extensional viscosity. In order to answer this question, virtual polymer melt $\mathrm{M}$ (having medium level of extensional strain hardening) has been chosen for FEM analysis for the case considering that downstream orifice die region is filled by the polymer melt. In this work, Compuplast software VEL 6.2 has been used together with the modified White-Metzner (mWM) constitutive equation according to Barnes and Roberts [10]. The sketch of the studied flow situation for conventional orifice die together with corresponding boundary conditions and FE mesh is provided in Figure 3 (grid consists of 7400 nodes and 3553 elements) whereas the Figure 4 describes the rheological behavior of the virtual polymer melt M. The problem has been solved for different flow rates and the obtained theoretical results were plotted in Figure 5 and compared with corresponding theoretical data considering that downstream orifice die region is not filled by the polymer melt. In this Figure, it is clearly visible that entrance viscosities (defined as entrance pressure drop divided by apparent shear rate) for both cases differ significantly within wide range of apparent shear rates. In more detail, the fact that the polymer melt fills the downstream region of the orifice die leads to significant entrance viscosity increase due to additional shear flow component occurring at this region. Clearly, such artificial entrance viscosity increase will leads to artificial uniaxial extensional viscosity increase calculated by using Cogswell [5], Binding [6] or Gibson model [7]. Therefore, the main conclusion from this theoretical study is that very narrow orifice downstream region may leads to erroneous estimation of the entrance viscosity leading to artificially high entrance pressure drop and uniaxial extensional viscosity. 

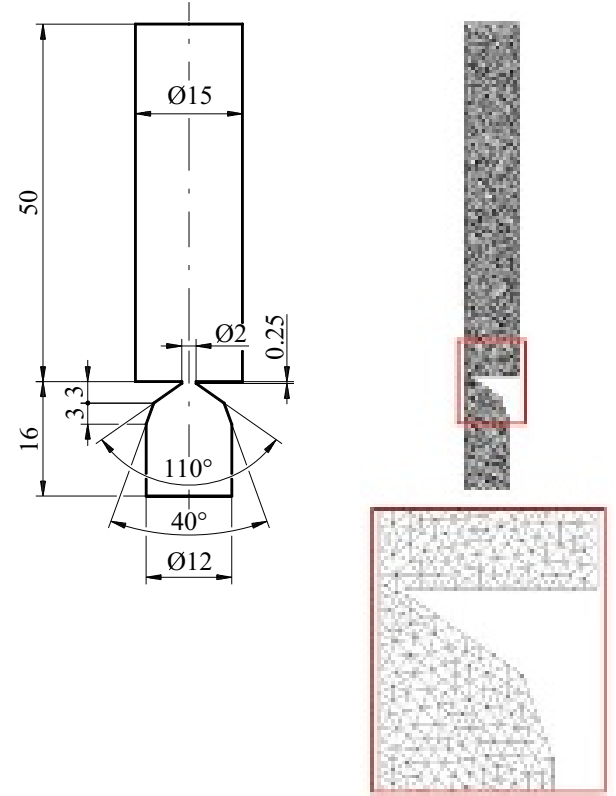

FIGURE 3. Conventional orifice die design.

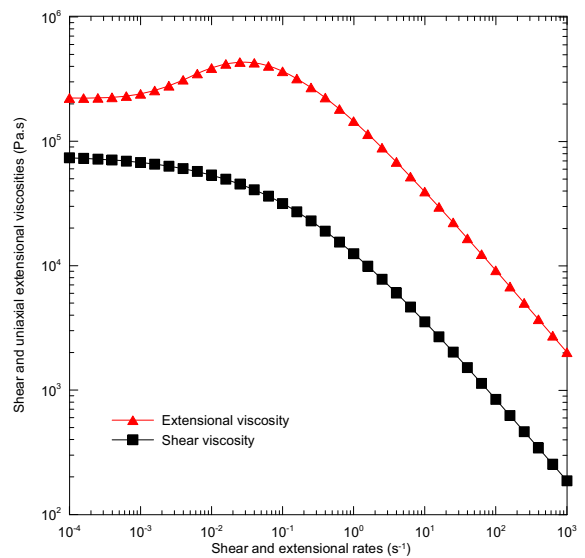

FIGURE 4. Rheological behavior of the virtual polymer melt $\mathrm{M}$ predicted by modified White-Metzner model [10].

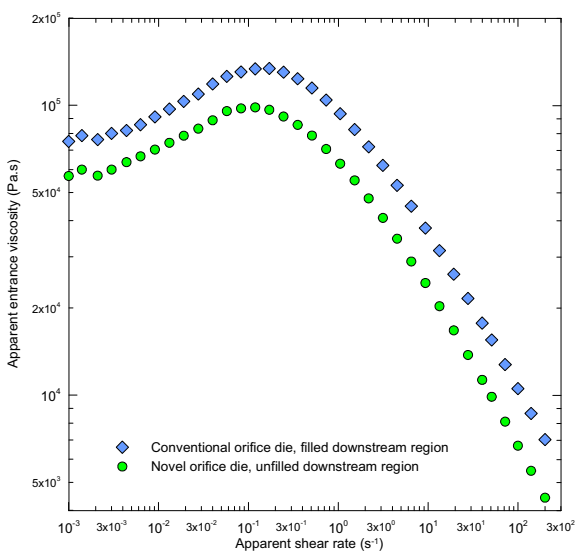

FIGURE 5. Theoretically predicted effect of die design (filled/unfilled orifice downstream region) on entrance viscosity (entrance pressure drop divided by apparent shear rate) for virtual polymer melt $\mathrm{M}$. 


\section{Novel Orifice Die Design}

In order to prevent unwanted artificial entrance viscosity increase due to very narrow downstream channel, novel patent pending orifice die design has been proposed. The sketch of the novel orifice die design is depicted in Figure 6.
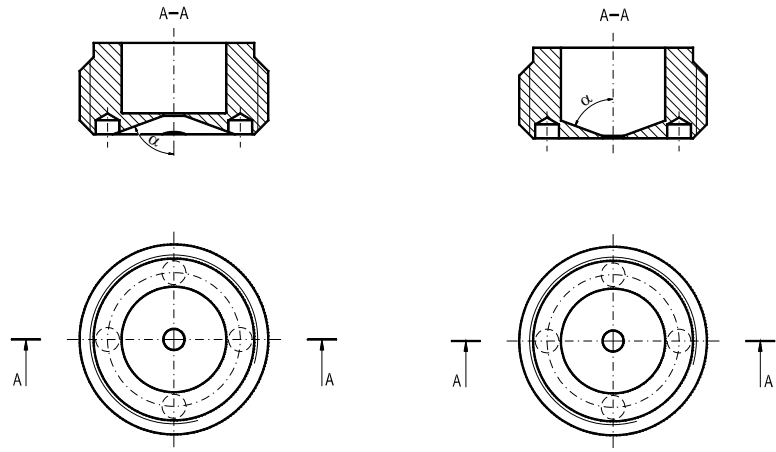

a)

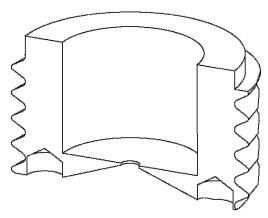

b)

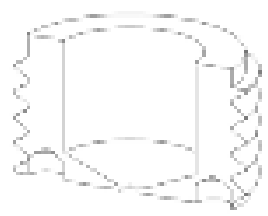

FIGURE 6. Novel patent pending orifice dies ( $\mathrm{a}$ - abrupt entry, $\mathrm{b}$ - convergent entry).

The main advantage of this novel orifice die design is occurrence of very open downstream region which consist of highly diverging channel (Figure 6a) or flat exit region (Figure 6b) and four holes which enables to use special key to crew-up the orifice die to the rheometer barrel. This downstream orifice die geometry eliminates any possibility for artificial pressure increase due to polymer melt touching the downstream wall. Moreover, possible detail view of the extrudate leaving the die during the measurements allow direct measurement of the free surface development during the extrudate swell which is practically impossible if one use the conventional orifice die design depicted in Figure 2. Another expected advantage of this novel die design can be much precise detection of the melt rupture for the rupture stress determination in comparison with conventional orifice design where the melt rupture can be hidden if downstream region is filled by the polymer melt. In the next part, this novel orifice die design will be tested for different processing conditions and polymer materials. 


\section{Experimental Evaluation of the Novel Orifice Die Design}

In order to evaluate the novel orifice die with respect to conventional orifice die, the entrance viscosity (entrance pressure drop divided by the apparent shear rate) has been measured for LDPE Lupolen $1840 \mathrm{H}$ at $250^{\circ} \mathrm{C}$ by using Rosand $\mathrm{RH} 7-2$ twin bore capillary rheometer and both orifice dies. The results together with the closer view of the orifice downstream region during measurements is provided in Figure 7.

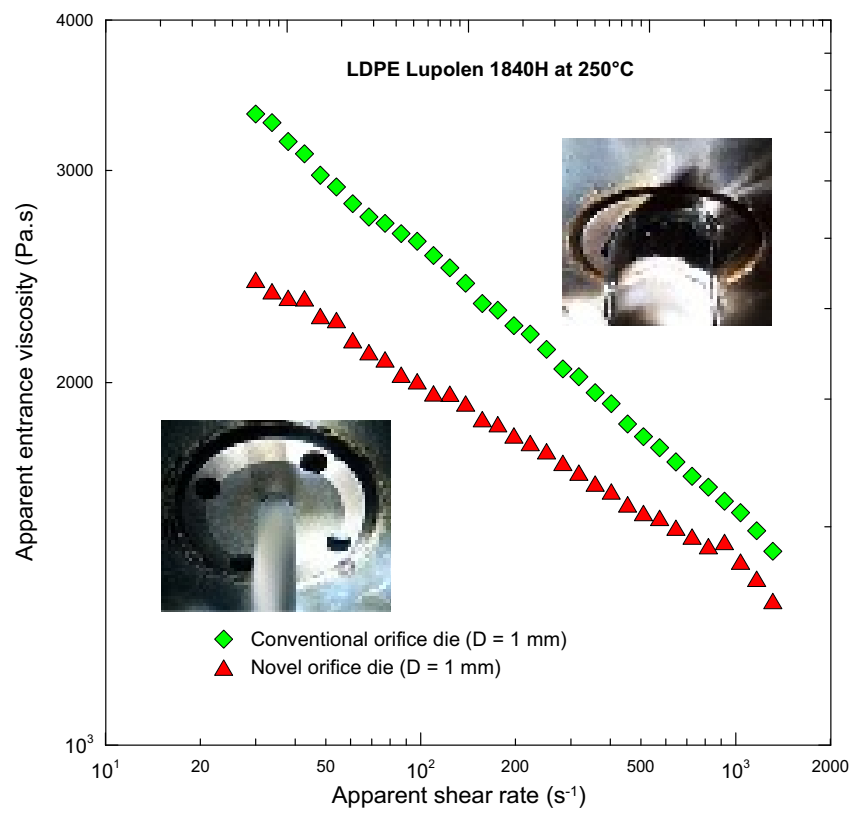

FIGURE 7. Comparison between experimentally determined entrance viscosities (entrance pressure drop divided by apparent shear rate) by using conventional orifice die and novel orifice die for LDPE Lupolen $1840 \mathrm{H}$ at $250^{\circ} \mathrm{C}$. The photographs represent closer view of the orifice downstream region during measurements for conventional orifice die design and novel patent pending orifice die design).

As expected from the theoretical study, entrance viscosity measured by the conventional orifice die is much higher in comparison with novel die design, clearly, due to the fact that polymer melt fills the downstream region of the conventional orifice die. Also, the level of the extrudate swell from the conventional orifice die is artificially high due to that reason. This leads us to the conclusion that the measurements of the extensional rheology by using conventional orifice die can be highly erroneous and thus, novel orifice die design should be preferred.

In the final step, novel orifice die has been used to determine extensional viscosity for mLLDPE Exact 0201 and HDPE Tipelin FS 450 - 26 film blowing grades and the obtained results were compared with experimental data obtained from Sentmanat extensional rheometer SER HV A01 (see Figure 8). As it can be clearly seen, 
the agreement between both experimentally determined extensional viscosities is very high suggesting that the use of the novel orifice die design can be used to very precise determination of the entrance pressure drop (at very low experimental error and very high experimental reproducibility) and consequently, by using proper technique, to valuable evaluation of the extensional viscosity of polymer melts.

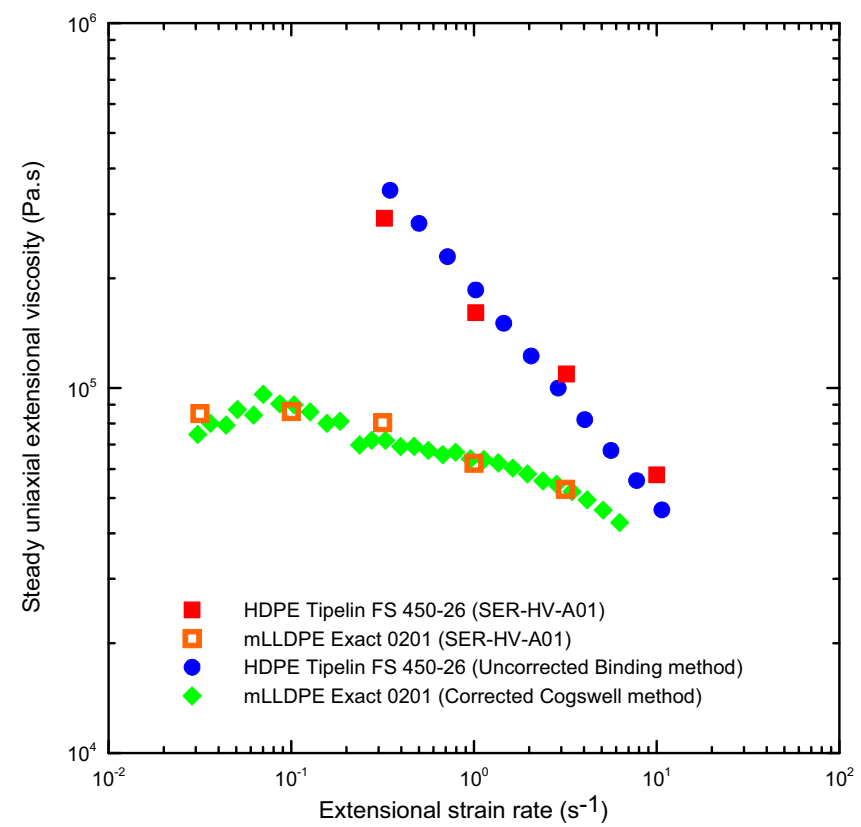

FIGURE 8. Comparison between extensional viscosity data obtained from entrance pressure drop measurements by using novel orifice die design and SER experimental data for mLLDPE Exact 0201 (corrected Cogswell model according to [9] has been used for extensional viscosity determination) and HDPE Tipelin FS 450-26 (uncorrected Binding model according to [6] has been used for extensional viscosity determination).

\section{CONCLUSION}

Novel patent pending orifice die design has been proposed and tested for the entrance pressure drop measurements. It has been theoretically and experimentally demonstrated that the novel orifice die design allows much precise entrance pressure drop measurement (and thus much precise extensional viscosity determination) in comparison with standard and conventional orifice dies. 


\section{ACKNOWLEDGMENTS}

The support of the projects by the Ministry of Education CR (KONTAKT ME08090, MSM 7088352101) is gratefully acknowledged.

\section{REFERENCES}

1. F. A. Morrison, Understanding rheology, New York: Oxford University press, Inc., 2001.

2. H. Münstedt, J. Rheol. 23, 421-436 (1979).

3. M. L. Sentmanat, Rheol. Acta 43, 657-669 (2004).

4. M. L. Sentmanat, B.N. Wang and G.H. McKinley, J. Rheol. 49 (3), 585-606 (2005).

5. F. N. Cogswell, Polym. Eng. Sci. 12, 64-73 (1972).

6. D. M. Binding, J. Non-Newtonian Fluid Mech. 27, 173-189 (1988).

7. A. G. Gibson, Composites 20, 57-64 (1989).

8. S. Kim and J. M. Dealy, J. Rheol. 45(6), 1413-1419 (2001).

9. M. Zatloukal, J. Vlcek, C. Tzoganakis and P. Saha, J. Non-Newtonian Fluid Mech. 107, 13-37 (2002).

10. H. A. Barnes and G. P. Roberts, J. Non-Newtonial Fluid Mech. 44, 113-126 (1992). 
Copyright of AIP Conference Proceedings is the property of American Institute of Physics and its content may not be copied or emailed to multiple sites or posted to a listserv without the copyright holder's express written permission. However, users may print, download, or email articles for individual use. 